\title{
Are Contraindications to Extracorporeal Membrane Oxygenation Slowly Vanishing?
}

In the mid-1980s, the application of extracorporeal membrane oxygenation (ECMO) in newborns with severe respiratory failure gained a footing when fewer than a dozen ECMO centers became established. What followed was the emergence of nearly 100 ECMO centers in the United States and very successful outcomes for neonates who would have probably died without ECMO. Inclusion and exclusion criteria were established, and ECMO became an important option in the care of newborns with severe respiratory failure unresponsive to conventional measures, which at the time was good old fashioned timecycled pressure-limited ventilation. ${ }^{1}$

In the early 1990s the use of ECMO in newborns began to decline with the introduction of high-frequency oscillatory ventilation, and continued to decline with the availability of surfactant replacement therapy and inhaled nitric oxide. $^{2}$ As a result of these advancements the treatment algorithm for respiratory failure in newborns was recalibrated, with ECMO used more in a rescue manner.

As centers gained more experience and ECMO systems improved, so did expanded applications, including pediatric and adult patients with respiratory and cardiac failure. Cardiac applications continue to increase, particularly in areas such as congenital heart disease and ECMO to aid resuscitation, known as ECMO-CPR. ${ }^{3}$ The hospital survival rates with these applications have not been as favorable as were those with the early neonatal experience, but the populations are more heterogeneous and include a wide range of diagnoses and comorbidities. Nonetheless, survival rates such as $50-60 \%$ for pediatric and adult respiratory failure and 30-40\% in ECMO-CPR support the use of ECMO beyond its proven niche. ${ }^{3-5}$

\section{See the Original Study on Page 941}

In this issue of RESPIRATORY CARE, Turner and colleagues add to the growing body of case series describing the successful use of ECMO for severe respiratory failure. ${ }^{6}$ They present 7 patients with profound respiratory failure associated with the 2009 H1N1 influenza pandemic and who were refractory to advanced therapies and ventilation strategies. Turner et al broach a number of important aspects of the decision making process for proceeding with
ECMO in critically ill patients when advanced clinical interventions fail, and their suggestion that "there are few absolute contraindications to ECMO" is intriguing. Historically, a key component in deciding to use ECMO has been the presumption that the disease process is irreversible. However, the reversible nature of respiratory failure associated with H1N1 was not well understood, so is an approach of we-won't-know-until-we-try reasonable?

Pre-ECMO ventilator duration beyond one week, and the presence of comorbidities have been deterrents for using ECMO, but these exclusion criteria were established for a homogenous group of neonates and in an era when lung-protective ventilation was not universally applied. In the case series the non-survivors had the longest pre-ECMO ventilator duration and were referred from outside hospitals. Turner et al underscore the importance of early transfer of patients with a worsening clinical trajectory so that ECMO remains a viable option, but they also suggest that the duration of mechanical ventilation alone may not be as important as the strategy applied. Perhaps the pre-ECMO ventilation duration should be relegated to the bottom of the ECMO contraindication list, assuming that lungprotective ventilation is applied.

The presence of comorbidities as exclusion criteria to ECMO continues to be deliberated. Death is often the alternative when ECMO is excluded as an option. The more experienced ECMO center may be willing to utilize ECMO in patients with chronic but stable conditions. For example, reports of ECMO for respiratory failure in patients with immune-compromised conditions have appeared in small case reports, albeit with fair outcomes. ${ }^{7}$ Turner et al provide a good argument for moving forward with ECMO in patients with underlying conditions. Maybe this should no longer be considered pushing the envelope.

An admirable survival rate of $71 \%$ is reported in the case series, ${ }^{6}$ and with relatively few complications, though not fully appreciated are the technical aspects such as the need for circuit troubleshooting. Interventions such as the replacement of components, air events, or circuit changes, particularly in the patient with a 37-day course, are not described, although the deaths in this series are not attributed to any technical complications. It seems that ECMO case reports are often focused on diagnoses, severity of illness, and outcome-all important, but we never 
really know how the ECMO course was managed from a technical perspective.

As H1N1 reached a pandemic threshold, reports of its prevalence in the southern hemisphere emerged with further accounts of an increased need for ECMO. Preparedness measures were being undertaken by hospitals with some questioning if ECMO should be included in their triage and treatment algorithms. Established ECMO centers wondered if they would be inundated with H1N1 cases, which could have overwhelmed existing resources.

Prior to the pandemic, ECMO centers began to make a welcomed change to the ECMO circuit by incorporating polymethylpentene oxygenators, ${ }^{8}$ such as described in the case series. ${ }^{6}$ These low-resistance devices are easy to prime and de-air, and are increasingly being used by many programs, and have simplified the conventional ECMO system. Turner et al also describe 2 different ECMO systems: one with a roller pump, and a newer centrifugal system. ${ }^{8}$ The transition from roller pump to centrifugal pump is occurring in many ECMO programs and is in part due to the need or perceived need for ECMO during the H1N1 outbreak.

As ECMO centers braced for an influx of potential H1N1 patients with presumed lengthy courses, they were faced with the possibility of insufficient personnel to "sit pump." Attention was then focused on creating more set-it-andforget-it systems that did not require round-the-clock vigilance by an ECMO specialist. Simplification has become a prominent theme in the ECMO community, with a paradigm shift toward ECMO systems with centrifugal pumps, minimal monitoring, and circuits with low prime volumes and few or no access points. ${ }^{9}$ The relative simplicity of some ventricular assist devices is often used as a comparison.

Establishing an ECMO program is an enormous undertaking, and doing so in the midst of a pandemic may present additional challenges, even with the availability of streamlined systems. The Extracorporeal Life Support Organization guidelines suggest that ECMO programs should manage a minimum of 6 cases annually, and provide ongoing education and emergency drills. ${ }^{5}$ Startup programs unable to meet these minimums may be faced with a steep learning curve and find it difficult to establish and maintain proficiency.

ECMO continues to be a vital life-saving option for critically ill patients with refractory respiratory failure. While the merits of ECMO have been strengthened in studies like the herculean CESAR (Conventional Ventilation or ECMO for Severe Adult Respiratory Failure) trial, we still do not-and probably never will- have a neat set of criteria to make the best possible decision about when to deploy ECMO. ${ }^{10}$ The case series by Turner et $\mathrm{al}^{6}$ highlights the current state of ECMO, further confirms its importance in the treatment algorithm for severe respiratory failure, and emphasizes the complexity of making the decision to use it.

\author{
Peter Betit RRT-NPS FAARC \\ Respiratory Care Department \\ and ECMO Program \\ Children's Hospital Boston \\ Harvard Medical School \\ Boston, Massachusetts
}

\section{REFERENCES}

1. Mugford M, Elbourne D, Field D. Extracorporeal membrane oxygenation for severe respiratory failure in newborn infants. Cochrane Data Syst Rev 2008;(3):CD001340. DOI:10.1002/14651858.

2. Fliman PJ, DeRegnier RO, Kinsella J, Reynolds M, Rankin LL, Steinhorn RH. Neonatal extracorporeal life support: impact on therapies on survival. J Pediatr 2006;148(5):595-599.

3. Thiagarajan RR, Laussen PC, Rycus PT, Bartlett RH, Bratton SL. Extracorporeal membrane oxygenation to cardiopulmonary resuscitation in infants and children. Circulation 2007;116(15):1693-1700.

4. Hemmila MR, Rowe SA, Boules TN, Miskulin L, McGillicuddy JW, Scheuer DJ, et al. Extracorporeal life support for severe acute respiratory distress syndrome in adults. Ann Surg 2004;240(4):595-607.

5. Extracorporeal Life Support Organization. Annual international registry and guidelines. http://www.elso.med.umich.edu/Registry.html. Accessed May 11, 2011.

6. Turner DA, Rehder KJ, Peterson-Carmichael SL, Ozment CP, AlHegelan MS, Williford WL, et al. Extracorporeal membrane oxygenation for severe refractory respiratory failure secondary to 2009 H1N1 influenza A. Respir Care 2011;56(7):941-946.

7. Gupta M, Stanley TP, Moler FW. Extracorporeal life support for severe respiratory failure in children with immune-compromised conditions. Pediatr Crit Care Med 2008;9(4):380-385.

8. Thiara APS, Noel TN, Kristiansen F, Karlsen HM, Fiane AE, Svenevig JL. Evaluation of oxygenators and centrifugal pumps for long-term pediatric extracorporeal membrane oxygenation. Perfusion 2007;22(5):323-326.

9. Palanzo D, Qiu F, Baer L, Clark JB, Myers JL, Undar A. Evolution of the extracorporeal life support circuitry. Artificial Organs 2010; 34(11):869-873. DOI: 10.1111/j.1525-1594.2010.01127.x.

10. Sidebotham D. Extracorporeal membrane oxygenation: understanding the evidence: CESAR and beyond. J Extra Copor Technol 2011; 43(1):P23-P26.

The author has disclosed no conflicts of interest.

Correspondence: Peter Betit RRT-NPS FAARC, Respiratory Care Department and ECMO Program, Children's Hospital Boston, MA-661, 300 Longwood Avenue, Boston MA 02115. E-mail: peter.betit@ childrens.harvard.edu.

DOI: $10.4187 /$ respcare. 01407 